\title{
Transfer of spin orientation into electric current in quantum wells
}

\author{
S.D. Ganichev ${ }^{\mathrm{a}, \mathrm{b}, *}$, E.L. Ivchenko ${ }^{\mathrm{b}}$, S.N. Danilov ${ }^{\mathrm{a}}$, M. Sollinger ${ }^{\mathrm{a}}$, J. Eroms $^{\mathrm{a}}$, \\ W. Wegscheider ${ }^{a, 1}$, D. Weiss ${ }^{\text {a }}$, W. Prettl ${ }^{\mathrm{a}}$ \\ ${ }^{a}$ Institut für Experimentelle und Angewandte Physik, Universität Regensburg, D-93040 Regensburg, Germany \\ b A.F. Ioffe Physico-Technical Institute of the RAS, 194021 St. Petersburg, Russia
}

\begin{abstract}
A monopolar spin polarization has been achieved in quantum well structures applying terahertz circularly polarized radiation. The spin polarization results in a directed motion of free carriers in the plane of a quantum well perpendicularly to the direction of light propagation. Due to spin selection rules the direction of the current is determined by the helicity of the light and can be reversed by switching the helicity from right- to left handed. (C) 2002 Elsevier Science B.V. All rights reserved.
\end{abstract}

Keywords: Circular photogalvanic effect; Monopolar spin polarization

A substantial portion of current research in condensed-matter physics is directed toward understanding various manifestations of spin-dependent phenomena. In particular, the spin of electrons and holes in solid state systems is the decisive ingredient for active spintronic devices [1,2] and several schemes of quantum computation [3-5]. Optical orientation by applying circularly polarized light is frequently used to prepare an ensemble of spin polarized carriers [6]. The experiments described below show that in low-dimensional systems with band splitting in $\boldsymbol{k}$-space due to $\boldsymbol{k}$-linear terms in the Hamiltonian optical excitation leads not only to a spin polarized ensemble of electrons but also to a current whose

\footnotetext{
* Corresponding author. Institut für Experimentelle und Angewandte Physik, Universität Regensburg, D-93040 Regensburg, Germany. Tel.: +49-941-943-2050; fax: +49-941-943-4223.

E-mail address: sergey.ganichev@physik.uni-regensburg.de (S.D. Ganichev).

${ }^{1}$ Also at: Walter Schottky Institut, TU München, 85748 Garching, Germany.
}

direction depends solely on the predominant spin orientation.

Here we report the conversion of free carrier spin polarization into an electric current for both n-type and p-type quantum wells. The observed spin photocurrent flows in the quantum well perpendicularly to the direction of the incident circularly polarized light. The current reverses its direction by switching the sign of helicity of the radiation and hence the spin orientation of free carriers. The effect is quite general and has been observed for all semiconductor systems investigated.

The experiments were carried out on heterostructures belonging to two different classes of symmetry. Higher symmetric structures were $\left(\begin{array}{lll}0 & 0 & 1\end{array}\right)$-MBE grown n-InAs/AlGaSb QWs with a $15 \mathrm{~nm}$ single InAs channel and (0 0101$)$-MBE grown n-GaAs/AlGaAs single heterojunctions. These structures can belong to two point groups, either $\mathrm{D}_{2 \mathrm{~d}}$ or $\mathrm{C}_{2 \mathrm{v}}$. Our measurements showed that all samples investigated here belong to the group $C_{2 v}$. Structures of the lower symmetry $\mathrm{C}_{\mathrm{s}}$ were (113)A-MBE grown p-GaAs/AlGaAs single QWs and multiple QWs (MQW) containing 20 
wells of $15 \mathrm{~nm}$ width. Samples of $\mathrm{n}$ - and $\mathrm{p}$-type with free-carrier densities between $10^{11}$ and $2 \times 10^{12} \mathrm{~cm}^{-2}$ were studied in the range from liquid helium to room temperature. Two pairs of ohmic contacts have been centered along opposite sample edges (see inset in Fig. 1). For optical excitation we used a high-power far-infrared pulsed $\mathrm{NH}_{3}$ laser optically pumped by a TEA $\mathrm{CO}_{2}$ laser which yields strong linearly polarized emission at wavelengths between 35 and $280 \mu \mathrm{m}$ [7]. The radiation induces indirect (Drude like) optical transitions in the lowest conduction subband of our n-type samples and direct optical transitions between valence subbands (heavy hole-light hole) in the p-type samples. Crystalline quartz $\lambda / 4$ plates allowed to modify the laser light polarization from linear to circular. The helicity $P_{\text {circ }}$ of the incident light varied from -1 (left-handed circular, $\sigma_{-}$) to +1 (right-handed circular, $\sigma_{+}$) according to $P_{\text {circ }}=\sin 2 \varphi$ where $\varphi$ is the angle between the initial plane of polarization and the optical axis of the $\lambda / 4$ plate.

In samples of the lower symmetry class $C_{s}$ grown on a (1 113$)$-GaAs surface, the spin photocurrent can be observed under normal incidence. The reversal of the current direction when the polarization switches from left-handed to right-handed circular is shown in Fig. 1 where the current is plotted as a function of the phase angle $\varphi$. For this symmetry the direction of current is determined by the symmetry of the crystal. The current flows always along the [1 10$]$-direction perpendicular to the plane of mirror reflection of the point group $\mathrm{C}_{\mathrm{s}}$, independent of the incidence plane of the

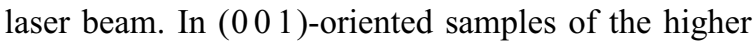
symmetry class $\mathrm{C}_{2 \mathrm{v}}$ a signal proportional to the helicity $P_{\text {circ }}$ is only observed under oblique incidence and the photocurrent is perpendicular to the wave vector of the incident light.

The solid line in Fig. 1 are obtained from a phenomenological picture outlined below which perfectly describes the experimental observations. The photogalvanic current under study can be described by the following expression:

$j_{\lambda}=\chi_{\lambda \mu \nu}\left(E_{\mu} E_{v}^{*}+E_{v} E_{\mu}^{*}\right) / 2+\gamma_{\lambda \mu} i\left(\boldsymbol{E} \times \boldsymbol{E}^{*}\right)_{\mu}$,

where $\boldsymbol{E}$ is the complex amplitude of the electric field of the electromagnetic wave and $i\left(\boldsymbol{E} \times \boldsymbol{E}^{*}\right)=$ $\hat{\mathbf{e}} P_{\text {circ }} E_{0}^{2}$, where $E_{0}=|\boldsymbol{E}|$ is the electric field amplitude and $\hat{\mathbf{e}}$ is the unit vector pointing in the direction of the light propagation. The photocurrent given by the

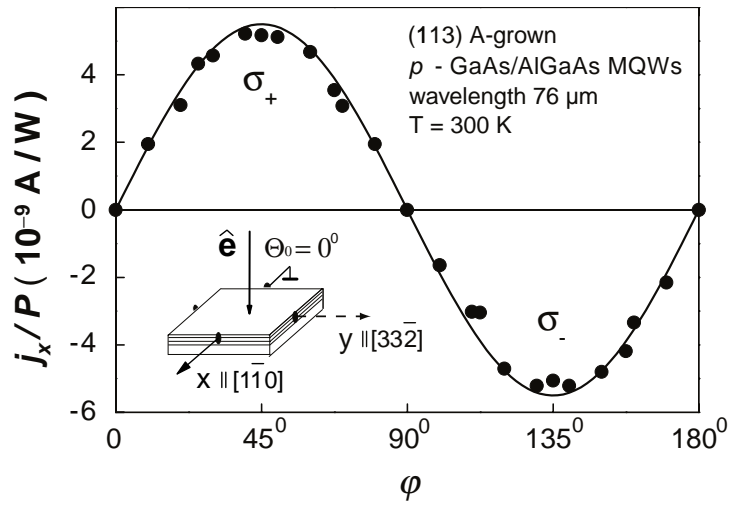

Fig. 1. Photocurrent in QWs normalized by the light power $P$ as a function of the phase angle $\varphi$ defining helicity. Full circles are experimental data and solid line is fitted using one parameter according to Eq. (3).

tensor $\chi$ describes the so-called linear photogalvanic effect (LPGE) [8] because it is usually observed under linearly polarized optical excitation. The circular photogalvanic effect (CPGE) described by the pseudotensor $\gamma$ can be observed only under circularly polarized excitation. The pseudotensor $\gamma$ can have nonzero components which depend on the symmetry of the system. For the higher symmetry $\mathrm{C}_{2 \mathrm{v}}$ applying for quantum well structures grown along the principal axis [0 01 1], a photocurrent can only be generated under oblique incidence of irradiation. For the point group $\mathrm{C}_{2 \mathrm{v}}$ the nonzero components of $\gamma$ are $\gamma_{x y}$ and $\gamma_{y x}$ with $x\left\|\left[\begin{array}{lll}1 & 1 & 0\end{array}\right], y\right\|\left[\begin{array}{lll}1 & 1 & 0\end{array}\right]$. If $(y, z)$ is the plane of incidence, then the photocurrent is induced along $x$ with

$j_{x}=\gamma_{x y} t_{\mathrm{p}} t_{\mathrm{s}} \sin \Theta E_{0}^{2} P_{\text {circ }}$,

where $E_{0}$ is the electric field amplitude in vacuum, $t_{\mathrm{p}}$ and $t_{\mathrm{s}}$ are transmission coefficients after Fresnel's formula for linear p- and s-polarizations, $\Theta$ is the angle of refraction defined by $\sin \Theta=\sin \Theta_{0} / n, \Theta_{0}$ is the angle of incidence and $n$ is the index of refraction. In the low-symmetry QWs grown along $z \|[h h l]$ with $[h h l] \neq\left[\begin{array}{lll}0 & 0 & 1\end{array}\right]$ or [ $\left[\begin{array}{lll}1 & 1 & 1\end{array}\right]$, the point group is $\mathrm{C}_{\mathrm{s}}$ which contains only two elements, the identity and one mirror reflection plane normal to the [1 10$]$-direction. In this case additionally the pseudotensor component $\gamma_{x z}$ is nonzero allowing the generation of a photocurrent along $x \|\left[\begin{array}{lll}1 & \overline{1} & 0\end{array}\right]$ also under normal incidence of radiation. The corresponding contribution to the 
photocurrent is

$j_{x}=\gamma_{x z} t_{\mathrm{p}} t_{\mathrm{s}} \cos \Theta E_{0}^{2} P_{\text {circ }}$.

The currents measured along $x$ as a function of the phase angle $\varphi$ (Fig. 1) and the angle of incidence $\Theta_{0}$ are in very good agreement with the phenomenological expressions Eq. (2) for symmetry $\mathrm{C}_{2 \mathrm{v}}$ and Eq. (3) for $\mathrm{C}_{\mathrm{s}}$. The figure shows experimental data compared to calculations which were fitted with one ordinate scaling parameter.

Microscopically a conversion of photon helicity into a spin photocurrent arises due to $\boldsymbol{k}$-linear terms in the effective Hamiltonian $H^{(1)}=\beta_{l m} \sigma_{l} k_{m}$ where $\boldsymbol{k}$ is the electron wave vector, $\sigma_{l}$ are the Pauli spin matrices and $\beta_{l m}$ are real coefficients. The coefficients $\beta_{l m}$ form a pseudotensor subjected to the same symmetry restriction as the pseudotensor $\gamma_{\lambda \mu}$. The coupling between $\sigma_{l}$ and the wave vector of the charge carriers $k_{m}$ as well as spin-controlled selection rules, described below, yield a net current dependent upon circularly polarized optical excitation. The effect is most easily conceivable for p-type materials from the schematic band structure shown in Fig. 2. For the sake of simplicity, we assume a band structure (two-dimensional dispersion) consisting only of the lowest conduction subband e1 and the highest heavy hole subband hh1. The $\boldsymbol{k}$-linear terms are taken into account only for the valence band and result in a splitting into two parabolas of different spin. In the case of $\mathrm{C}_{\mathrm{s}}$ symmetry the splitting is given by $E_{\mathrm{hh} 1, \pm 3 / 2}(\boldsymbol{k})=-\left(\hbar^{2} k^{2} / 2 m_{h}\right) \pm \beta k_{x}{ }^{2}$.

We consider direct optical transitions which, depending on the photon energy, may be of interband or intersubband type (heavy hole-light hole or heavy hole-heavy hole subbands in p-type materials). The theoretical concept of the model is the same for both types of transitions [9]. However, for intersubband transitions the $\boldsymbol{k}$-linear terms, which are different for different bands and subbands, have to be taken into account for both subbands involved and complicate the picture. Hence, we concentrate on the interband excitations shown in Fig. 2. While not directly illustrating the transitions in our p-type QWs, Fig. 2 makes the point more transparent than a discussion of the more complex intersubband transitions in the valence band.

\footnotetext{
${ }^{2}$ The case of the $\mathrm{C}_{2 \mathrm{v}}$ symmetry can be considered in a similar way. For the $\mathrm{C}_{\mathrm{s}}$ symmetry described here the coordinate system

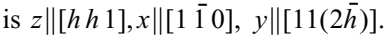

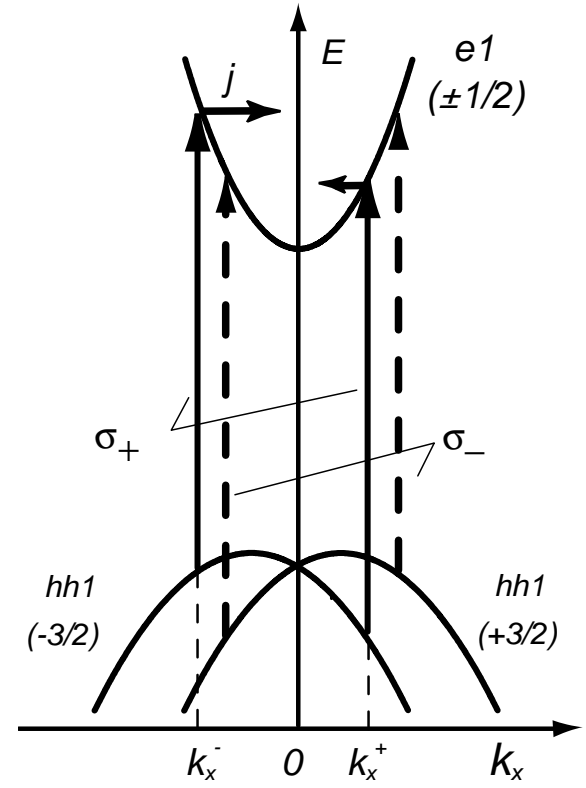

Fig. 2. Microscopic picture describing the origin of spin polarized photocurrents. The essential ingredient is the splitting of the valence band due to $\boldsymbol{k}$-linear terms. $\sigma_{+}$excitation induces direct transitions (solid arrows) from $\mathrm{s}=-3 / 2(\mathrm{hh} 1)$ to $-1 / 2(\mathrm{e} 1)$ with unbalanced occupation of the positive $k_{x}$ and negative $k_{x}$ states resulting in a spin polarized photocurrent. For $\sigma_{-}$excitation (dashed arrows) both the spin orientation of the charge carriers and the current direction get reversed.

Qualitatively, the results are the same. In Fig. 2, the allowed optical transitions are from $\mathrm{s}=-3 / 2$ to $-1 / 2$ for the $\sigma_{+}$photon polarization and from $\mathrm{s}=3 / 2$ to $1 / 2$ for the $\sigma_{-}$polarization. The s are the spin quantum numbers of the corresponding electron states. Under circularly polarized radiation with a photon energy $\hbar \omega$ and for fixed value of $k_{y}$ energy and momentum conservation allow transitions only for two values of $k_{x}$. For the $\sigma_{+}$photons these $k_{x}$ values of photogenerated electrons are

$$
\begin{aligned}
k_{x}^{ \pm}= & -\frac{\mu}{\hbar^{2}} \beta \pm\left[\frac{2 \mu}{\hbar^{2}}\left(\hbar \omega-E_{\mathrm{g}}\right)-k_{y}^{2}\right. \\
& \left.+\left(\frac{\mu}{\hbar^{2}} \beta\right)^{2}\right]^{-1 / 2},
\end{aligned}
$$

where $E_{\mathrm{g}}$ is the energy gap, $m_{\mathrm{e}, \mathrm{h}}$ are the effective masses of electrons and holes, respectively, and $\mu$ is the reduced electron-hole effective mass $m_{\mathrm{e}} m_{\mathrm{h}} /\left(m_{\mathrm{e}}+\right.$ $\left.m_{\mathrm{h}}\right)$. The corresponding transitions are shown in Fig. 2 
by the solid vertical arrows with their center of 'mass' shifted from the point $k_{x}=0$ by $-\beta\left(\mu / \hbar^{2}\right)$. Thus, the sum of the electron velocities in the excited states, $\hbar\left(k_{x}^{+}+k_{x}^{-}\right) / m_{\mathrm{e}}=-2\left(\mu / m_{\mathrm{c}}\right)(\beta / \hbar)$, is nonzero and the contribution of $k_{x}^{ \pm}$photoelectrons to the current do not cancel as in the case $\beta=0$. Consequently, a spin polarized net current in the $x$-direction results. Changing the photon helicity from +1 to -1 inverts the current because the 'center of mass' of these transitions is now shifted to $+\beta\left(\mu / \hbar^{2}\right)$ (see dashed arrows in Fig. 2).

In summary, the experiments carried out on different types of quantum wells have shown that circularly polarized light can generate a directed electric current at room temperature. The microscopic picture given above requires that the generated current is spin polarized and suggests that the system can be considered as a source for spin polarized currents. The effect, being sensitive to the degree of spin orientation, provides an easy access to spin dynamics in semiconductor structures [10]. We emphasize that in gyrotropic media with $\boldsymbol{k}$-linear terms in the Hamiltonian, spin injection yielding an imbalance of spin orientation leads always to a current. As quantum wells based on III-V compounds are gyrotropic, spin polarization causes in any case a current flow.
The high quality InAs quantum wells were kindly provided by J. De Boeck and G. Borghs from IMEC Belgium. The technical support of M. Bichler is gratefully acknowledged. We also acknowledge financial support from the DFG, the RFFI and the NATO linkage program.

\section{References}

[1] S. Datta, B. Das, Appl. Phys. Lett. 56 (1990) 665.

[2] G.A. Prinz, Phys. Today 48 (1995) 58.

[3] B.E. Kane, Nature 393 (1998) 133.

[4] D. Loss, D.P. DiVincenzo, Phys. Rev. A 57 (1998) 120.

[5] D.P. DiVincenzo, D. Bacon, J. Kempe, G. Burkard, K.B. Whaley, Nature 408 (2000) 339.

[6] F. Meier, B.P. Zakharchenya (Eds.), Optical Orientation, Elsevier Science Publ., Amsterdam, 1984.

[7] S.D. Ganichev, Physica B 273-274 (1999) 737.

[8] S.D. Ganichev, H. Ketterl, W. Prettl, E.L. Ivchenko, L.E. Vorobjev, Appl. Phys. Lett. 77 (2000) 3146.

[9] S.D. Ganichev, E.L. Ivchenko, S.N. Danilov, J. Eroms, W. Wegscheider, D. Weiss, W. Prettl, Phys. Rev. Lett. 86 (2001) 4358

[10] S.D. Ganichev, S.N. Danilov, V.V. Bel'kov, E.L. Ivchenko, M. Bichler, W. Wegscheider, D. Weiss, W. Prettl, cond-mat/0107466, 2001. 